\title{
Chatting in Distributed Quantization Networks
}

\author{
John Z. Sun and Vivek K Goyal \\ Research Laboratory of Electronics \\ Massachusetts Institute of Technology \\ johnsun@mit.edu, v.goyaleieee.org
}

\begin{abstract}
Several key results in source coding offer the intuition that distributed encoding via vector-quantize-and-bin is only slightly suboptimal to joint encoding and oftentimes is just as good. However, when source acquisition requires the blocklength to be small, collaboration between sensors can greatly reduce distortion. For a distributed acquisition network where sensors are allowed to "chat" using a side channel, we provide exact characterization of distortion performance and quantizer design in the high-resolution (low-distortion) regime using a framework called distributed functional scalar quantization (DFSQ). The key result is that chatting can dramatically improve performance even when the intersensor communication is at very low rate. We also solve the rate allocation problem when communication links have heterogeneous costs and provide examples to demonstrate that this theory predicts performance at practical communication rates.
\end{abstract}

\section{INTRODUCTION}

The Slepian-Wolf theorem shows that for lossless communication of correlated information sources, distributed encoding can perform as well as joint encoding [1]. Although this surprising and beautiful result does not extend fully, comparable results for lossy coding show that the rate loss can be small using Berger-Tung encoding (see, e.g., [2]), again suggesting that communication between encoders of correlated information sources has little or no utility. However, the Slepian-Wolf result is dependent on large blocklength; in the finite-blocklength regime, the optimality of distributed encoding does not hold [3].

This paper examines the use of communication among encoders when the blocklength of acquisition and quantization is 1. The Slepian-Wolf intuition-that communication between encoders does not help-is not valid. Instead, collaboration among encoders, called chatting in this work, can greatly decrease the aggregate communication from the encoders to the decoder to meet a distortion criterion. We explore chatting by building upon the distributed functional scalar quantization (DFSQ) framework, which explores compression through scalar quantization when the decoder desires fidelity in computing a function of the sources [4], [5]. Specifically, we consider the architecture shown in Fig. 1, where $N$ correlated but memoryless continuousvalued, discrete-time stochastic processes produce scalar realizations $X_{1}^{N}(t)=\left(X_{1}(t), \ldots, X_{N}(t)\right)$ for $t \in \mathbb{Z}$. For each $t$, realizations of these sources are scalar quantized by

This material is based upon work supported by the National Science Foundation under Grant No. 1115159.

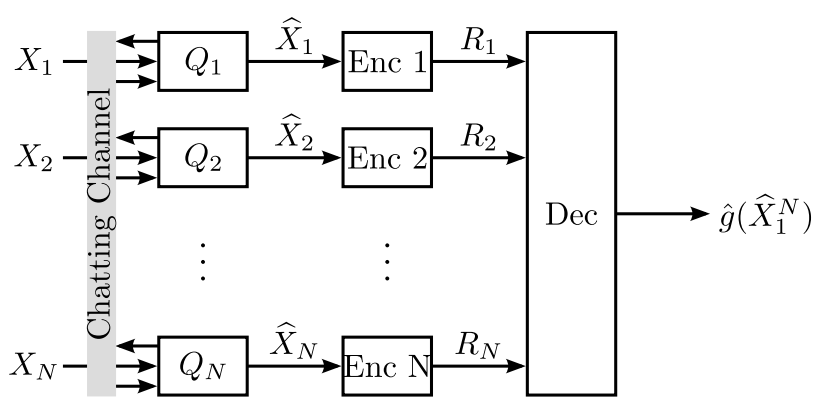

Fig. 1. A distributed computation network, where $N$ sensors (comprising quantizer and encoder) observe realizations of correlated sources. Each observation $X_{n}$ is encoded and communicated over rate-limited links to a central decoder. Simultaneously, each sensor can interact with a subset of the other sensors using a noiseless but rate-limited chatting channel to improve compressibility. The decoder computes an estimate of the function $g\left(X_{1}^{n}\right)=g\left(X_{1}, X_{2}, \ldots, X_{n}\right)$ from the received data using a reconstruction function $\hat{g}\left(\widehat{X}_{1}^{n}\right)$ but cannot observe messages communicated on the chatting channel.

sensors and transmitted to a central decoder at rates $R_{1}^{N}$. Concurrently, sensors can collaborate with each other via a side channel that is unobservable to the decoder. Since the quantization is scalar and the source is memoryless, we remove the time index and model the sources as being draw iid from a joint distribution $f_{X_{1}^{N}}$ at each $t$.

The main contributions of this paper are to precisely characterize the distortion performance of a distributed network when chatting is allowed and determine the optimal quantizer design for each sensor. In this setting, we show that collaboration can have significant impact on performance; in some cases, it can dramatically reduce distortion even when the chatting has extremely low rate. Since the decoder desires a computation of the source realizations, effective chatting reveals information about both the conditional distribution of the source a sensor is acquiring and how important the source measurement is to the decoder. We analyze the dual nature of chatting messages and draw qualitative observations about their design. We also give necessary conditions on the chatting topology and protocol for successful decodability in the DFSQ framework, and find the optimal rate allocation when links are heterogeneous and have different costs of transmission.

This work has recently been extended in [6]. 


\section{Preliminaries}

\section{A. Previous Work}

There is a large body of literature studying performance of the distributed network in Fig. 1 without the chatting channel; a comprehensive review of these works and their connections to DFSQ appears in [4]. Similarly, connections to coding for computing (e.g. [7], [8]) are discussed there as well.

Here, we review results that relate to the chatting channel, focusing on Shannon-theoretic results. Kaspi and Berger provided inner bounds for the rate region of a two-encoder problem where one encoder can send information for the other using compress-and-forward techniques [9]. Recently, this bound has been generalized in [10], but the exact rate region is still unknown except in special cases. Chatting is related to source coding problems such as interaction [11], [12], omniscience [13] and data exchange [14]. However, these settings are more naturally suited for discrete-alphabet sources and existing results rely on large-blocklength analysis.

There are also connections between this work and distortion side information [15] and vector quantization with alternative distortion measures [16].

\section{B. Distributed Functional Scalar Quantization}

We emphasize that the focus of this work is on compression of continuous-valued sources using small blocks of data. Here, performance results from Shannon theory are overly optimistic since tools such as joint-typicality encoding and decoding are not reliable. Instead, we consider the complementary asymptotic of high resolution, where the blocklength is considered fixed and the compression rate $R$ is made large [17], [18]. We now introduce high-resolution theory and summarize key results from DFSQ [4], [5].

1) Quantization basics: A scalar quantizer $Q_{K}$ is a mapping from the real line to a set of $K$ points $\mathcal{C}=\left\{c_{k}\right\}_{k=1}^{K} \subset \mathbb{R}$ called the codebook, where $Q_{K}(x)=c_{k}$ if $x \in P_{k}$ and the cells $\left\{P_{k}\right\}_{k=1}^{K}$ form a partition of $\mathbb{R}$. The quantizer is called regular if the partition cells are intervals containing the corresponding codewords. We then assume the codebook entries are indexed from smallest to largest and that $P_{k}=\left(p_{k-1}, p_{k}\right]$ for each $k$, implying $p_{0}<c_{1} \leq p_{1}<c_{2} \leq \cdots<c_{K} \leq p_{K}$, with $p_{0}=-\infty$ and $p_{K}=\infty$. Define the granular region as $\left(c_{1}, c_{K}\right)$ and its complement $\left(-\infty, c_{1}\right] \cup\left[c_{K}, \infty\right)$ as the overload region.

Uniform quantization, where partition cells in the granular region have equal length, is most commonly used in practice, but nonuniform quantization can provide significant performance improvements. Fig. 2 presents the compander model as a method for generating nonuniform quantizers from a uniform one, where the scalar source is transformed using a nondecreasing and smooth compressor function $c: \mathbb{R} \rightarrow[0,1]$, then quantized using a uniform quantizer $Q_{K, U}$ comprising $K$ levels on the granular region [0,1], and finally passed through the expander function $c^{-1}$. Compressor functions are defined such that $\lim _{x \rightarrow-\infty} c(x)=0$ and $\lim _{x \rightarrow \infty} c(x)=1$. It is convenient to define a point density

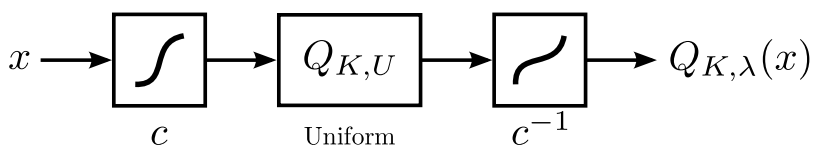

Fig. 2. A block diagram for companding as a constructive method for nonuniform scalar quantization. The notation $Q_{K, U}$ is used to describe the canonical uniform quantizer with $K$ partitions in the granular region $[0,1]$.

function as $\lambda(x)=c^{\prime}(x)$. Because of the extremal conditions on $c$, there is a one-to-one correspondence between $\lambda$ and $c$, and hence a quantizer of the form shown in Fig. 2 can be uniquely specified using a point density function and codebook size. We denote such a quantizer as $Q_{K, \lambda}$.

2) High-resolution theory: It is generally difficult to compute the distortion of a scalar quantizer for a given $K$. However, the distortion behavior of $Q_{K, \lambda}$ can be precisely analyzed as the number of codewords $K$ becomes large. Assuming the source is well-modeled as being drawn iid from a probabilistic distribution, we define the mean squared error (MSE) distortion as

$$
D_{\text {mse }}(K, \lambda)=\mathrm{E}\left[\left|X-Q_{K, \lambda}(X)\right|^{2}\right],
$$

where the expectation is with respect to the source density $f_{X}$. Under the additional assumption that $f_{X}$ is continuous (or simply measurable) with tails that decay sufficiently fast,

$$
D_{\text {mse }}(K, \lambda) \simeq \frac{1}{12 K^{2}} \mathrm{E}\left[\lambda^{-2}(X)\right]
$$

where $\simeq$ indicates that the ratio of the two expressions approaches 1 as $K$ increases [19], [20]. Hence, the MSE performance of a scalar quantizer can be approximated by a simple relationship between the source distribution, point density and codebook size, and this relation becomes more precise with increasing $K$. Moreover, quantizers designed according to this approximation are asymptotically optimal, meaning that the quantizer optimized over $\lambda$ has distortion that approaches the performance of the best $Q_{K}$ found by any means [21]-[23]. Experimentally, the approximation is surprisingly accurate even for moderate $K$ [24], [25]. Since the distortion's dependence on the shape of the quantizer $\lambda$ is explicit in the asymptote, calculus techniques can be used to optimize companders.

When the quantized values are to be communicated or stored, it is natural to map each codeword to a string of bits and consider the trade-off between performance and communication rate $R$, defined to be the expected number of bits per sample. In the simplest case, the codewords are indexed with equal-length labels and the communication rate is $R=\log _{2}(K)$; this is called fixed-rate or codebookconstrained quantization. Hölder's inequality can be used to show that the optimal point density for fixed-rate is asymptotically

$$
\lambda_{\mathrm{mse}, \mathrm{fr}}^{*}(x) \propto f_{X}^{1 / 3}(x),
$$

and the resulting distortion is

$$
D_{\mathrm{mse}, \mathrm{fr}}^{*}(R) \simeq \frac{1}{12}\left\|f_{X}\right\|_{1 / 3} 2^{-2 R},
$$


with the notation $\|f\|_{p}=\left(\int_{-\infty}^{\infty} f^{p}(x) d x\right)^{1 / p}$ [26]. The limit conditions on $c(x)$ imply the integral of $\lambda(x)$ is unity; we hide the normalization when presenting point density results for clarity.

In general, the codeword indices can be coded to produce bit strings of different lengths based on probabilities of occurrence; this is referred to as variable-rate quantization. If the decoding latency is allowed to be large, one can employ block entropy coding and the communication rate approaches $H\left(Q_{K, \lambda}(X)\right)$. This particular scenario, called entropy-constrained quantization, can be analyzed using Jensen's inequality to show the optimal point density $\lambda_{\text {mse,ec }}^{*}$ is asymptotically constant on the support of the input distribution [26]. The optimal quantizer is uniform and the resulting distortion is

$$
D_{\mathrm{mse}, \mathrm{ec}}^{*}(R) \simeq \frac{1}{12} 2^{-2(R-h(X))} .
$$

Note that block entropy coding suggests that the sources are transmitted in blocks even though the quantization is scalar. As such, (5) is an asymptotic result and serves as a lower bound on practical entropy coders with finite blocklengths that match the latency restrictions of a network.

3) Distributed functional scalar quantization: In a distributed network where the sensors employ scalar quantization and the decoder performs a known computation, optimizing for the computation rather than source fidelity can lead to substantial gains. For DFSQ, the distortion criterion is functional MSE (fMSE):

$$
D_{\text {fmse }}\left(K_{1}^{N}, \lambda_{1}^{N}\right)=\mathrm{E}\left[\left|g\left(X_{1}^{N}\right)-\hat{g}\left(Q_{K_{1}^{N}, \lambda_{1}^{N}}\left(X_{1}^{N}\right)\right)\right|^{2}\right],
$$

where $g$ is a scalar function of interest, $\hat{g}$ is the decoding function and $Q_{K_{1}^{N}, \lambda_{1}^{N}}$ is scalar quantization performed on a vector such that

$$
Q_{K_{1}^{N}, \lambda_{1}^{N}}\left(x_{1}^{N}\right)=\left(Q_{\lambda_{1}, K_{1}}\left(x_{1}\right), \ldots Q_{\lambda_{N}, K_{N}}\left(x_{N}\right)\right) .
$$

Before understanding how a quantizer affects fMSE, it is convenient to define how a computation locally affects distortion.

Definition 2.1 ([4]): The nth functional sensitivity profile of a multivariate function $g$ is defined as

$$
\gamma_{n}(x)=\left(\mathrm{E}\left[\left|g_{n}\left(X_{1}^{N}\right)\right|^{2} \mid X_{n}=x\right]\right)^{1 / 2},
$$

where $g_{n}(x)$ is the partial derivative of $g$ with respect to its $n$th argument evaluated at the point $x$.

Given the sensitivity profile, the main result of DFSQ [4] is

$$
D_{\text {fmse }}\left(K_{1}^{N}, \lambda_{1}^{N}\right) \simeq \sum_{n=1}^{N} \frac{1}{12 K_{n}^{2}} \mathrm{E}\left[\left(\frac{\gamma_{n}\left(X_{n}\right)}{\lambda_{n}\left(X_{n}\right)}\right)^{2}\right],
$$

with conditional expectation decoder

$$
\hat{g}\left(x_{1}^{N}\right)=\mathrm{E}\left[g\left(X_{1}^{N}\right) \mid Q_{K_{1}^{N}, \lambda_{1}^{N}}\left(X_{1}^{N}\right)=Q_{K_{1}^{N}, \lambda_{1}^{N}}\left(x_{1}^{N}\right)\right],
$$

provided the following conditions are satisfied:
MF1. The function $g$ is Lipschitz continuous and twice differentiable in every argument except possibly on a set of Jordan measure 0 .

MF2. The source pdf $f_{X_{1}^{N}}$ is continuous, bounded, and supported on $[0,1]^{N}$.

MF3. The function $g$ and set of point densities $\lambda_{1}^{N}$ allow $\mathrm{E}\left[\left(\gamma_{n}\left(X_{n}\right) / \lambda_{n}\left(X_{n}\right)\right)^{2}\right]$ to be defined and finite for all $n$.

Similar conditions are given in [5] for infinite-support distributions and a simpler decoder.

Following the same recipes to optimize over $\lambda_{1}^{N}$ as in the MSE setting, the relationship between distortion and communication rate is found. In both cases, the sensitivity acts to shift quantization points to where they can reduce the distortion in the computation. For fixed rate, the distortion approximated by (8) is minimized by

$$
\lambda_{n, \mathrm{fmse}, \mathrm{fr}}^{*}(x) \propto\left(\gamma_{n}(x) f_{X_{n}}(x)\right)^{1 / 3},
$$

where $f_{X_{n}}$ is the marginal distribution of $X_{n}$. In the entropyconstrained case, the optimal point density is

$$
\lambda_{n, \mathrm{fmse}, \mathrm{ec}}^{*}(x) \propto \gamma_{n}(x) .
$$

4) Don't-care intervals: When the computation induces the sensitivity to be 0 on some intervals of the support, the high-resolution assumptions are violated and the asymptotic distortion performance may not be described by (8). This issue is addressed by carefully coding when the source is in such a "don't-care" interval [4, Sec. VII]. This consideration is particularly relevant because chatting can often induce the sensitivity to be 0 , and proper coding can lead to greatly improved performance.

Consider $L_{n}$ don't-care intervals in $\gamma_{n}$ and let $A_{n}$ be the event that the source realization is not in the unions of them. In the fixed-rate setting, one codeword is allocated to each don't-care interval, and the remaining $K_{n}-L_{n}$ codewords are used to form reconstruction points in the nonzero intervals. There is a small degradation in performance from the loss corresponding to $L_{n}$, but this quickly becomes negligible as $K_{n}$ increases. In the entropy-constrained case, the additional flexibility in coding allows for the encoder to split its message and reduce cost. The first part is an indicator variable revealing whether the source is not in a don't-care interval and can be coded at rate $I_{A} \equiv H_{B}\left(P\left(A_{n}\right)\right)$, where $H_{B}$ is the binary entropy function. The actual reconstruction message is only sent if event $A_{n}$ occurs and its rate is amplified to $\left(R_{n}-I_{A}\right) / P\left(A_{n}\right)$ to meet the average rate constraint. The multiplicative factor $1 / P\left(A_{n}\right)$ is called the rate amplification.

5) Chatting: In [4, Sec. VIII], chatting is introduced in the setting where one sensor sends exactly one bit to another sensor. Under fixed-rate quantization, this collaboration can at most decrease the distortion by a factor of 4 using a property of $\mathcal{L}_{1 / 3}$ quasi-norms. Because utilizing that bit to send additional information to the decoder would decrease distortion by exactly a factor of 4 , this is considered a negative result. Here, there is an implicit assumption that links have equal cost per bit and the network wishes to 
optimize a total cost budget. In the entropy-constrained setting, chatting may be useful even when links have equal costs. One example was given to demonstrate a single bit of chatting can decrease the distortion by an unbounded amount; more generally, the benefits of chatting varies depending on the source joint distribution and decoder computation.

In previous work, there is no systematic theory on performance and quantizer design of chatting. Moreover, collaboration in larger networks was still an open problem. In this paper, we extend previous results and provide a more complete discussion on how a chatting channel affects a distributed acquisition network.

\section{Performance And Design of Chatting NETWORKS}

We model the chatting channel in Fig. 1 as a directed graph $\mathcal{G}^{c}=(\mathcal{V}, \mathcal{E})$, where the set of nodes $\mathcal{V}$ is the set of all sensors and $\mathcal{E} \subseteq \mathcal{V} \times \mathcal{V}$ is the set of noiseless, directed chatting links. The parent and children sets of a sensor $n \in \mathcal{V}$ are denoted $\mathcal{N}_{p}(n)$ and $\mathcal{N}_{c}(n)$ respectively. If $(i, n) \in \mathcal{E}$, then for each source realization sensor $i$ sends to sensor $n$ a chatting message $M_{i \rightarrow n}^{c}$ with rate $R_{i \rightarrow n}^{c}$. For convenience, the set of chatting messages is denoted $M^{c}$ and the set of corresponding link rates are $R^{c}$. The chatting messages $M_{i \rightarrow n}^{c}$ are communicated according to a schedule that the sensors and the central decoder know in advance. We assume chatting occurs quickly in that it is completed before the next discrete time instant (at which point new realizations of $X_{1}^{N}$ are sensed). After chatting is complete, each sensor $n$ compresses its quantized observation $\widehat{X}_{n}$ into a message $M_{n}$, which is communicated to the decoder at rate $R_{n}$.

We now present fMSE performance of $Q_{K_{1}^{N}, \lambda_{1}^{N}}$ in the fixed-rate and entropy-constrained settings, and show how to optimize $\lambda_{1}^{N}$ given rates $R_{1}^{N}$ and $R^{c}$ imposed by network constraints. We first provide analysis assuming the decoder can can successfully recover the quantized values from messages $M_{1}^{N}$. Later in Section III-C, we provide conditions on the chatting graph $\mathcal{G}^{c}$ and set of chatting messages $M^{c}$ such that the decoder is successful with zero error, having benefited from already understanding the quantizer design.

Before analyzing distortion, we need to extend the idea of functional sensitivity. Recall that $\mathcal{N}_{p}(n) \subseteq V$ is the set of parents of sensor $n$ in the chatting graph $\mathcal{G}^{c}$.

Definition 3.1: The $n$th conditional sensitivity profile of computation $g$ given all chatting messages $M^{c}$ is

$$
\begin{array}{r}
\gamma_{n \mid M^{c}}(x \mid m)=\left(\mathrm { E } \left[\left|g_{n}\left(X_{1}^{N}\right)\right|^{2} \mid X_{n}=x,\right.\right. \\
\left.\left.M_{i \rightarrow n}^{c}=m_{i \rightarrow n} \forall i \in \mathcal{N}_{p}(n)\right]\right)^{1 / 2} .
\end{array}
$$

Notice only messages from parent sensors are relevant to $\gamma_{n \mid M^{c}}$. Intuitively, chatting messages reveal information about the parent sensors' quantized values and reshape the sensitivity appropriately. Depending on the encoding of chatting messages, this may induce don't-care intervals in the conditional sensitivity (where $\gamma_{n \mid M^{c}}=0$ ).
The distortion dependence on the number of codeword points and the conditional sensitivity profiles is given in the following theorem:

Theorem 3.2: Given the source distribution $f_{X_{1}^{N}}$, computation $g$, and point densities $\lambda_{1}^{N}\left(M^{c}\right)$ satisfying conditions MF1-3 for every $M^{c}$, the distortion performance given codeword allocation $K_{1}^{N}$ and $K^{c}$ is asymptotically

$$
\begin{aligned}
& D_{\mathrm{fmse}}\left(K_{1}^{N}, K^{c}, \lambda_{1}^{N}\right) \\
& \simeq \mathrm{E}\left[\sum_{n=1}^{N} \mathrm{E}_{X_{n} \mid M^{c}}\left[\frac{1}{12 K_{n}^{2}(m)} \frac{\gamma_{n \mid M^{c}}^{2}\left(X_{n} \mid m\right)}{\lambda_{n \mid M^{c}}^{2}\left(X_{n} \mid m\right)} \mid M^{c}=m\right]\right] .
\end{aligned}
$$

We assume the decoder is the conditional expectation decoder in (9).

Proof: The proof mirrors the proof of (8), but requires refinements based on Theorems 17 in [4].

Following the techniques in [5], the theorem can be expanded to account for infinite-support distributions and a simpler decoder. Using this result, we can now generalize quantizer design with chatting.

\section{A. Fixed-rate Quantization Design}

We mirror the method used to determine (10) in the standard DFSQ setup but must adjust the quantizer depending on the incoming messages. As a result, a sensor chooses from a set of codebooks corresponding to the conditional sensitivity induced by incoming messages from parent sensors. Let $Z_{n}\left(M^{c}\right)$ be the union of the don't-care intervals induced by chatting messages $M^{c}$. Then the optimal point density for fixed-rate quantization is

$$
\begin{aligned}
& \lambda_{n, \mathrm{fmse}, \mathrm{fr}, \text { chat }}^{*}(x \mid m) \\
& \propto\left\{\begin{array}{l}
\left(\gamma_{n \mid M^{c}}(x \mid m) f_{X_{n} \mid M^{c}}(x \mid m)\right)^{1 / 3}, \\
x \notin Z_{n}(m) \text { and } f_{X_{n} \mid M^{c}}(x \mid m)>0 ; \\
0, \quad \text { o.w. }
\end{array}\right.
\end{aligned}
$$

Recall that the point density is the derivative of the compressor function $c(x)$ in the compander model. More discussion on converting point densities into finite-codeword quantizers is given in [5]. Note that one codeword must be placed in each don't-care interval, as discussed previously in Section II-B.

\section{B. Entropy-constrained Quantization Design}

In the entropy-constrained case, the optimal point density is

$$
\begin{aligned}
& \lambda_{n, \text { fmse,ec,chat }}^{*}(x \mid m) \\
& \propto\left\{\begin{array}{l}
\gamma_{n \mid M^{c}}(x \mid m), \\
x \notin Z_{n}(m) \text { and } f_{X_{n} \mid M^{c}}(x \mid m)>0 ; \\
0, \quad \text { o.w. }
\end{array}\right.
\end{aligned}
$$

Here, even more care is needed in incorporating don't-care intervals, following the strategy discussed in Section II-B. Note that rate amplification can arise through chatting, and this can allow distortion terms to decay at rates faster than $2^{-2 R_{n}}$. 


\section{Conditions on Chatting}

We observe that chatting can influence optimal design of scalar quantizers through the conditional sensitivity, and the sensors will vary the quantization codebook $\mathcal{C}_{n}$ depending on the incoming messages from parent sensors. Hence, success of compression is contingent on the central decoder identifying the codebook employed by every sensor from the messages $M_{1}^{N}$.

Definition 3.3: A chatting network is codebook identifiable if the decoder can determine the codebooks of $Q_{K_{1}^{N}, \lambda_{1}^{N}}$ using the messages it receives from each sensor. That is, it can determine $\mathcal{C}_{n}\left(M^{c}\right)$ from $M_{1}^{N}$ for each time instant.

We have argued that a chatting network can successfully communicate its compressed observations if it is codebook identifiable. The following are sufficient conditions on the chatting graph $\mathcal{G}^{c}$ and messages $M^{c}$ such that the network is codebook identifiable:

$\mathrm{C} 1$. The chatting graph $\mathcal{G}^{c}$ is a directed acyclic graph.

$\mathrm{C} 2$. The causality in the chatting schedule matches $\mathcal{G}^{c}$, meaning sensor $n$ sends its chatting message after its parents and before its children.

C3. The quantizer at sensor $n$ is a function of the source joint distribution and all incoming messages from parent sensors in $N_{p}(n)$.

C4. The messages emanating from sensor $n$ is a function of $M_{n}$ and incoming messages from parent sensors in $N_{p}(n)$.

When each sensor's quantizer is regular and encoder only operates on the quantized values $\widehat{X}_{n}$, matching the DFSQ setup, these conditions are necessary as well. In this setting, the chatting message can only influence the choice of codebook; more complicated acquisition structure such as information fusion and coordination at the sensor are beyond the scope of this paper.

\section{Rate Allocation in Heterogeneous Networks}

A strong motivation for intersensor chatting is that sensors may be geographically closer to each other than a base station and hence require less transmit power. Moreover, low-bandwidth orthogonal channels may exist but are not utilized in a distributed network. As a result, the cost of communicating a bit may vary in a network; this work implies that there are worthwhile performance gains if chatting can be implemented on low-bandwidth, low-cost links. In this section, we investigate the optimal rate allocation for compression in a distributed network with heterogeneous links given a total cost constraint.

We define the cost per bit of a communication link between sensor $n$ and the decoder to be $\alpha_{n}$, and the corresponding cost allocation to be $b_{n}$. Similarly, for a chatting link between sensors $i$ and $n$, the cost per bit and cost allocation are defined as $\alpha_{i \rightarrow n}^{c}$ and $b_{i \rightarrow n}^{c}$ respectively. Being consistent with previous notation, we denote $b^{c}$ as the set of cost allocations on chatting links. Given a total $\operatorname{cost} C$, an acquisition network yields a distortion-cost tradeoff that can optimized.

Definition 4.1: The operational distortion-cost function of a distributed network with a set of chatting links $\mathcal{E}$ is defined as the least distortion possible from $N$ scalar quantizers with total cost $C$ or less:

$$
\delta(C) \equiv \inf _{\sum_{n=1}^{N} b_{n}+\sum_{(i, n) \in \mathcal{E}}^{N}, b^{c}, \lambda_{1 \rightarrow n}^{N}:} D_{\text {fmse }}\left(b_{1}^{N}, b^{c}, \lambda_{1}^{N}\right) .
$$

The operational distortion-cost function is a lower bound on functional distortion achievable with scalar quantizers of a given cost; it is difficult to determine analytically or even experimentally in the chatting framework. However, we can utilize high-resolution analysis, understanding that it is asymptotically optimal and provides meaningful approximation at lower rates. Below, we solve the cost allocation problem of communication links given chatting allocation $b^{c}$ and the distortion formula of Theorem 3.2.

Lemma 4.2: The optimal solution to

$$
D\left(C^{\prime}\right)=\min _{\sum b_{n}=C^{\prime}, b_{n} \geq 0} \sum_{n=1}^{N} \beta_{n} 2^{-2 b_{n} / \alpha_{n}}
$$

has cost allocation

$$
b_{n}^{*}=\max \left(0, \frac{1}{2} \log _{2} \frac{\beta_{n} / \alpha_{n}}{\bar{\beta}}\right),
$$

where $\bar{\beta}$ is chosen such that $\sum b_{n}^{*}=C^{\prime}$.

Proof: This follows from [27] or can be derived from the KKT conditions.

The above lemma yields the cost allocation $b_{1}^{N}$ where

$$
C^{\prime}=C-\sum_{(i, n) \in \mathcal{E}} b_{i \rightarrow n}^{c} .
$$

Optimization over chatting allocations in full generality is difficult but may not be realistic as network constraints may limit the maximum chatting rate, especially when the link cost may be small. In the examples presented later, we assume the rates are fixed for all chatting links.

When the solution in Lemma 4.2 is strictly positive, it can be expressed in closed form:

Lemma 4.3: Assuming the optimal solution to (16) has costs that are strictly positive, the optimizing costs are

$$
b_{n}^{*}=\frac{\alpha_{n}}{\sum \alpha_{i}} C^{\prime}+\frac{\alpha_{n}}{2} \log _{2} \frac{\beta_{n} / \alpha_{n}}{\left(\prod_{j}\left(\beta_{j} / \alpha_{j}\right)^{\alpha_{j}}\right)^{1 / \sum \alpha_{i}}} .
$$

Proof: The proof uses Lagrangian optimization and joint solution of the following two equations:

$$
\begin{aligned}
b_{n}^{*} & =-\frac{\alpha_{n}}{2} \log _{2} \lambda-\frac{\alpha_{n}}{2} \log _{2} \frac{\alpha_{n}}{2 \beta_{n} \ln 2} \\
\log _{2} \lambda & =-\frac{2 C^{\prime}}{\sum \alpha_{i}}-\frac{1}{\sum \alpha_{i}} \sum_{j=1}^{N} \alpha_{j} \log _{2} \frac{\alpha_{j}}{2 \beta_{j} \ln 2} .
\end{aligned}
$$

If a sensor is allowed to vary the communication rate with the chatting messages it receives, a more refined cost allocation can be utilized. This situation is natural in the entropy-constrained setting, and we will demonstrate later 
that this generalization can yield rate amplification. The distortion-cost function is:

$$
D\left(C^{\prime}\right)=\min _{\substack{\sum \mathrm{E}\left[b_{n}\left(M^{c}\right)\right]=C^{\prime} \\ b_{n}(m) \geq 0}} \sum_{n=1}^{N} \mathrm{E}\left[\beta_{n}\left(M^{c}\right) 2^{-2 b_{n}\left(M^{c}\right) / \alpha_{n}}\right] .
$$

Hence, each link will have an allocation $b_{n}\left(M^{c}\right)$ for every possible $M^{c}$ while satisfying an average cost constraint. An analogous result to Lemma 4.3 can be determined but we omit the closed form for brevity.

\section{EXAMPLES}

We now present examples to demonstrate when chatting can improve distortion performance and provide intuition for system design. The main goal is to illuminate the conditional sensitivity in (12) and the cost allocation lemmas.

\section{A. Independent Sources, Separable Computations}

First we give sufficient conditions under which quantizer design, and hence performance, does not change with chatting. Define a separable function $g$ as

$$
g\left(X_{1}^{N}\right)=\sum_{n=1}^{N} g^{(n)}\left(X_{n}\right)
$$

where $g^{(n)}$ is a scalar-parameter function. In separable functions, the partial derivatives $g_{n}$ only depend on $X_{n}$ and the sensitivity cannot be changed by chatting messages. For independent sources, chatting is similarly irrelevant since the conditional joint distribution is the same as the joint distribution. Thus, for independent sources and separable computations, each sensor's quantizer does not vary with chatting, and distortion remains the same. Examples of this include mean and sum calculations on iid sources.

\section{B. Correlated Sources with Linear Computation}

In standard DFSQ theory, correlations among the sources are only useful in an aggregate sense for computing the sensitivity, and specific realizations of one source cannot affect the acquisition of another. However, the scheduling implicit in chatting networks allows the sensor to use its neighbors' acquisitions as side information, and knowledge of the conditional joint distribution can change and refine the shape of the conditional sensitivity. Here, we demonstrate that chatting can decrease distortion in linear computations, which is not the case in standard DFSQ theory.

Denote $U(a, b)$ as the uniform distribution on support $[a, b]$. Consider $X_{1}$ and $X_{2}$ such that $X_{1} \sim \mathcal{U}(0,1)$ and

$$
X_{2} \sim \begin{cases}\mathcal{U}(0,1 / 2), & X_{1} \in[0,1 / 2] \\ \mathcal{U}(1 / 2,1), & \text { o.w. }\end{cases}
$$

For a computation $g\left(X_{1}, X_{2}\right)=\eta_{1} X_{1}+\eta_{1} X_{2}$, DFSQ without chatting yields distortion

$$
\frac{\eta_{1}}{12} 2^{-2 R_{1}}+\frac{\eta_{2}}{12} 2^{-2 R_{2}}
$$

for both the fixed-rate and entropy-constrained settings.

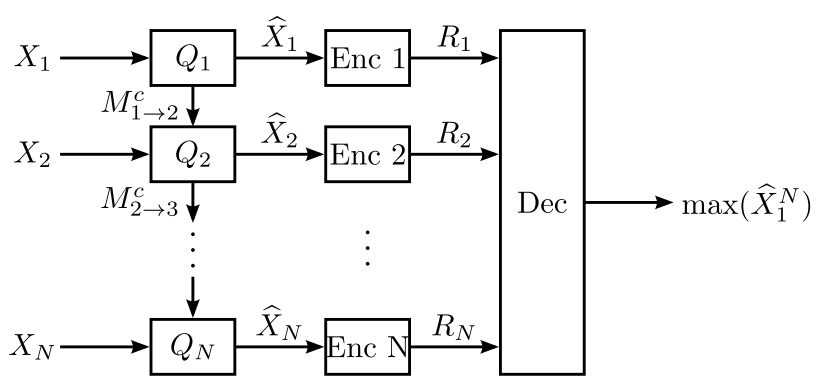

Fig. 3. A decoder wishes to determine the maximum of $N$ iid uniform sources. The sensors are allowed to chat serially down the network using a message $M_{(n-1) \rightarrow n}^{c}$ at rate $R_{c}$.

Meanwhile, a single bit of communication $M_{1 \rightarrow 2}^{c}$ improves performance. Taking into account coding over don't-care intervals but not optimizing rate allocations, the fixed-rate setting will yield distortion

$$
\frac{\eta_{1}}{12} 2^{-2 R_{1}}+\frac{\eta_{2}}{24} 2^{-2 R_{2}}
$$

Similarly, entropy-constrained quantization provides even better performance:

$$
\frac{\eta_{1}}{12} 2^{-2 R_{1}}+\frac{\eta_{2}}{24} 2^{-4\left(R_{2}-1\right)} .
$$

\section{Independent Sources, Maximum Computation}

We now consider a more complicated task, which is to reproduce the maximum of $N$ sources at the decoder with chatting in cascade (Fig. 3). To simplify the analysis, each source is $X_{n} \sim \mathcal{U}(0,1)$ iid. We also simplify the chatting channel by assuming the following:

1) The chatting is serial, meaning the set of chatting messages is $\left\{M_{(n-1) \rightarrow n}^{c}\right\}_{n=2}^{N}$.

2) Each chatting link is identical and has rate $R_{c}$, codebook size $K_{c}=2^{R_{c}}$ and cost $\alpha_{c}$.

3) The chatting message at sensor 1 is a uniformly quantized version of its observation.

4) For $n>1$, the chatting message is the maximum of its own uniformly quantized observation and the incoming chatting message.

Under this architecture, we rewrite the chatting as

$$
M_{(n-1) \rightarrow n}^{c}=\mathcal{I}\left(Q_{K_{c}, U}\left(\max \left(X_{1}^{n-1}\right)\right)\right),
$$

where $\mathcal{I}$ is the index of the quantization codeword and can takes values $\left\{1, \ldots, K_{c}\right\}$. Hence, the chatting message into sensor $n$ is a coarse quantization of the maximum of all ancestor sensors.

From (7), the max function has sensitivity $\gamma_{n}^{2}(x)=x^{N-1}$. Without chatting, each sensor's quantizer would be the same, utilizing the source distribution and the sensitivity. With chatting, we also need to determine the conditional sensitivity, which is given below for uniform sources: 
Lemma 5.1: Given $K_{c}=2^{R_{c}}$, the sensitivity profile given chatting message $M_{(n-1) \rightarrow n}^{c}=k$ is

$$
\begin{aligned}
& \gamma_{n \mid M_{(n-1) \rightarrow n}^{c}(x \mid k)}^{2} \\
& \quad= \begin{cases}0, & x<\frac{k-1}{K_{c}} ; \\
\frac{\left(K_{c} x\right)^{n-1}-(k-1)^{n-1}}{k^{n-1}-(k-1)^{n-1}} x^{N-n}, & \frac{k-1}{K_{c}} \leq x<\frac{k}{K_{c}} ; \\
x^{N-n}, & x \geq \frac{k}{K_{c}} .\end{cases}
\end{aligned}
$$

Proof: See Appendix A.

We note the incident chatting message of sensor $n$ is a uniformly quantized observation of $\max \left(X_{1}^{n-1}\right)$, with distribution $f(x)=(n-1) x^{n-2}$. Hence,

$$
\mathrm{P}\left(M_{(n-1) \rightarrow n}^{c}=k\right)=\left(\frac{k}{K_{c}}\right)^{n-1}-\left(\frac{k-1}{K_{c}}\right)^{n-1} .
$$

Below, we give performance bounds on both fixed-rate and entropy-constrained quantization schemes.

1) Fixed-rate case: From high-resolution theory, the distortion contribution from sensor $n$ is

$$
D_{n} 2^{-2 R_{n}},
$$

where $D_{n}=\frac{1}{12}\left\|\gamma_{n}^{2}\right\|_{1 / 3}$. Because $\gamma_{1}^{2}(x)=x^{N-1}$, the resulting distortion term (21) is

$$
D_{1}=\frac{1}{12}\left(\frac{3}{N+2}\right)^{3} \text {. }
$$

For other sensors, the distortion contribution is

$$
D_{n}=\frac{1}{12} \sum_{k=1}^{K_{c}} \mathrm{P}\left(M_{(n-1) \rightarrow n}^{c}=k\right)\left\|\gamma_{n \mid M_{(n-1) \rightarrow n}^{c}}^{2}=k\right\|_{1 / 3} .
$$

All messages besides $k=1$ induce a don't-care interval, so one of the $2^{R_{n}}$ codewords is placed exactly at $(k-1) / K_{c}$. This leads to some suboptimality relative to (21) which becomes negligible quickly as $R_{n}$ increases.

The tradeoff between chatting rate $R_{c}$ and fMSE is shown in Fig. 4 for several choices of $N$ and $\alpha_{c}$. We emphasize these results have optimal cost allocation determined by Lemma 4.3. For a finite total cost constraint corresponding to approximately $4 \mathrm{bits} / \mathrm{sample}$, sequences of practical quantizers are generated and yield similar performance to the asymptotic distortion performance of Theorem 3.2.

2) Entropy-constrained case: Generally, the distortion contribution from sensor $n$ in the entropy-constrained case is still (21), but must take into account the don't-care intervals that arise. Hence, the distortion constants are

$$
\begin{aligned}
D_{n, k}=\frac{1}{12} \mathrm{P} & \left(M_{(n-1) \rightarrow n}^{c}=k\right) \mathrm{P}\left(A_{n, k}\right) . \\
& \left.2^{2 h\left(X_{n} \mid A_{n, k}\right)+2 \mathrm{E}\left[\log _{2} \gamma_{n} \mid M_{(n-1) \rightarrow n}^{c}\right.}\left(X_{n} \mid k\right) \mid A_{n, k}\right]
\end{aligned}
$$

where $A_{n, k}$ is the event that the source realization of $X_{n}$ is not in a don't-care region of $\gamma_{n \mid M_{(n-1) \rightarrow n}^{c}}\left(X_{n} \mid k\right)$. As discussed previously, the message $M_{n}$ first codes an indicator variable corresponding to whether the source realization is in a don't-care interval, and then codes the codeword with rate $\left(R_{n}-H_{B}\left(P\left(A_{n, k}\right)\right)\right) / P\left(A_{n, k}\right)$ when relevant.

The tradeoff between chatting rate $R_{c}$ and fMSE is shown in Fig. 4, utilizing the cost allocation optimization in (19).

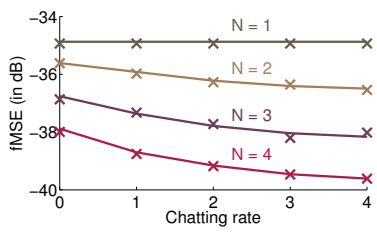

(a)

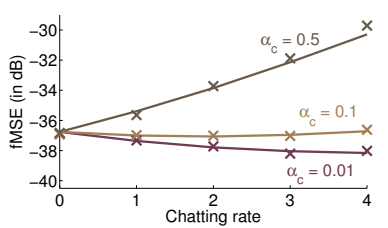

(c)

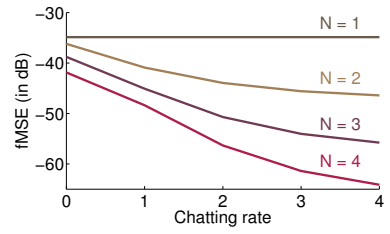

(b)

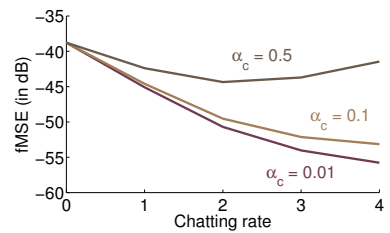

(d)
Fig. 4. Performance of the maximum computation network in both the fixed-rate (left plots) and entropy-constrained (right plots) settings. Plots (a) and (b) illustrate the tradeoff between fMSE and chatting rate for choices of $N$ assuming total cost $C=4 N$ and $\alpha_{c}=0.01$. Plots (c) and (d) illustrate the tradeoff between fMSE and chatting rate for choices of $\alpha_{c}$ assuming $N=4$ sensors and total cost $C=4 N$. In all cases, the cost of communication is $\alpha_{n}=1$. For the fixed-rate setting, we validate the distortion through simulated runs on real quantizers designed using (13). We observe that high-resolution theory predicts actual performance at rates as low as 4 bits/sample, as shown by crosses in the fixed-rate plots.

\section{Qualitative Messages}

Since distortion performance and cost allocation are so dependent on the source distribution, computation, and chatting graph, the general formulation may not be illuminating. Therefore, examples are essential in forming intuition about the effects of chatting. Below, we compile some key observations about chatting and its role in distributed compression:

1) Chatting messages affect sensitivity by allowing the sensor to leverage source realizations acquired at parent sensors to form a better quantizer using the conditional joint distribution, as shown in the second example, or to have a better understanding of whether its measurement is meaningful at the decoder, like in the third example. The dual nature of chatting is extremely interesting because the two goals may be in conflict with each other.

2) When the function is separable, chatting should only serve to aid in formation of a more informative conditional joint distribution. When the sources are independent, chatting should reveal how important a sensor's acquisition is to the decoder's computation. When the function is separable and the sources are independent, chatting is irrelevant.

3) When all links in the network have the same cost per bit, chatting may only be useful in the entropy-constrained case. When the cost of chatting is low relative to communication, chatting can be universally useful. However, the relationship between fMSE and chatting rate may be complicated and not always monotonic.

4) Cost allocation can greatly improve performance, especially when don't-care regions arise from chatting. The optimal cost allocations may be very different even though the communication links have similar cost per bit. When a sensor can adapt its communication rate to 
the decoder depending on the messages it receives, the use of (19) yields even greater gains.

\section{CONCLUSIONS}

In this work, we generalize the concept of intersensor interaction in a distributed acquisition network constrained to scalar quantization. We have motivated the DFSQ framework from two directions: the optimistic intuition offered by Shannon theory that becomes inapplicable when blocklength is small, and the potential gains over simplistic designs. There is great opportunity to leverage heterogeneous network design in information acquisition utilizing the tools of highresolution theory, and we provide precise characterizations of distortion performance, quantizer design, and cost allocation to optimize distributed networks.

Many challenges remain in analyzing chatting networks. Some future directions that are meaningful include more refined optimization of cost allocation, a more systematic understanding of how to design chatting messages, and theory on how chatting scales in large networks. One can consider "sensors" being distributed in time rather than space, with the decoder computing a function of samples from a random process. Connections of this formulation to structured vector quantizers are of independent interest.

\section{APPENDIX A \\ PROOF OF LEMMA 5.1}

The sensitivity of the maximum computation network assuming iid uniform sources is

$$
\begin{aligned}
\gamma_{n}^{2}(x) & =\mathrm{E}\left[\left|g_{n}\left(X_{1}^{N}\right)\right|^{2} \mid X_{n}=x\right] \\
& =\mathrm{P}\left(\max \left(X_{1}^{N}\right)=X_{n} \mid X_{n}=x\right) \\
& =\mathrm{P}\left(X_{1}<x\right) \cdots \mathrm{P}\left(X_{n-1}<x\right) . \\
& \quad \mathrm{P}\left(X_{n+1}<x\right) \cdots \mathrm{P}\left(X_{N}<x\right) \\
& =x^{N-1} .
\end{aligned}
$$

With chatting, sensor $n$ has some lossy version of the information collected by ancestor sensors. Defining $K_{c}=$ $2^{R_{c}}$ and denoting the received message $M_{(n-1) \rightarrow n}^{c}$ as $k_{n}$, there are now three distinct intervals of the sensitivity. First, if $x<\left(k_{n}-1\right) / K_{c}, X_{n}$ is less than $\max \left(X_{1}^{n-1}\right)$ and hence sensitivity is 0 since the information at sensor $n$ is irrelevant at the decoder. If $x>k_{n} / K_{c}, X_{n}$ is greater than $\max \left(X_{1}^{n-1}\right)$ and the sensitivity should only depend on the number of descendant sensors, leading to a sensitivity of $x^{N-n}$. Finally, when $\left(k_{n}-1\right) / K_{c} \leq x<k_{n} / K_{c}$, sensor $n$ must take into consideration both ancestors and descendants. First, we must find the effect of the parents:

$$
\begin{aligned}
\mathrm{P}\left(\max \left(X_{1}^{n-1}\right)<x\right. & \left.M_{(n-1) \rightarrow n}=k_{n}\right) \\
= & \frac{x^{n-1}-\left(\left(k_{n}-1\right) / K_{c}\right)^{n-1}}{k_{n} / K_{c}^{n-1}-\left(\left(k_{n}-1\right) / K_{c}\right)^{n-1}} .
\end{aligned}
$$

Next, we consider the descendants: $\mathrm{P}\left(\max \left(X_{n+1}^{N}\right)<x\right)=$ $x^{N-n}$. The product of the probabilities gives the final form.

\section{REFERENCES}

[1] D. Slepian and J. K. Wolf, "Noiseless coding of correlated information sources," IEEE Trans. Inform. Theory, vol. IT-19, pp. 471-480, July 1973.

[2] R. Zamir, "The rate loss in the Wyner-Ziv problem," IEEE Trans. Inform. Theory, vol. 42, pp. 2073-2084, Nov. 1996.

[3] V. Y. F. Tan and O. Kosut, "On the dispersions of three network information theory problems." arXiv:1201.3901v2 [cs.IT]., Feb. 2012.

[4] V. Misra, V. K. Goyal, and L. R. Varshney, "Distributed scalar quantization for computing: High-resolution analysis and extensions," IEEE Trans. Inform. Theory, vol. 57, pp. 5298-5325, Aug. 2011.

[5] J. Z. Sun, V. Misra, and V. K. Goyal, "Distributed functional scalar quantization simplified." arXiv:1206.1299v1 [cs.IT]., June 2012.

[6] J. Z. Sun and V. K. Goyal, "Collaboration in distributed quantization networks." Submitted, Oct. 2012.

[7] A. Orlitsky and J. R. Roche, "Coding for computing," IEEE Trans. Inform. Theory, vol. 47, pp. 903-917, Mar. 2001.

[8] H. Feng, M. Effros, and S. A. Savari, "Functional source coding for networks with receiver side information," in Proc. 42nd Annu. Allerton Conf. Commun. Control Comput., pp. 1419-1427, Sept. 2004.

[9] A. H. Kaspi and T. Berger, "Rate-distortion for correlated sources with partially separated encoders," IEEE Trans. Inform. Theory, vol. IT-28, pp. 828-840, Nov. 1982.

[10] M. Sefidgaran and A. Tchamkerten, "On cooperation in multi-terminal computation and rate distortion," in Proc. IEEE Int. Symp. Inform. Theory, pp. 771-775, July 2012.

[11] N. Ma and P. Ishwar, "Some results on distributed source coding for interactive function computation," IEEE Trans. Inform. Theory, vol. 57, pp. 6180-6195, Sept. 2011.

[12] N. Ma, P. Ishwar, and P. Gupta, "Interactive source coding for function computation in collocated networks," IEEE Trans. Inform. Theory, vol. 58, pp. 4289-4305, July 2012.

[13] S. Nitinawarat and P. Narayan, "Perfect omniscience, perfect secrecy, and Steiner tree packing," IEEE Trans. Inform. Theory, vol. 56, pp. 6490-6500, Dec. 2010.

[14] T. Courtade and R. Wesel, "Efficient universal recovery in broadcast networks," in Proc. 48th Аnnu. Allerton Conf. Commun. Control Comput., pp. 1542-1549, Oct. 2010.

[15] E. Martinian, G. W. Wornell, and R. Zamir, "Source coding with distortion side information," IEEE Trans. Inform. Theory, vol. 54, pp. 4638-4665, Oct. 2008.

[16] T. Linder, R. Zamir, and K. Zeger, "High-resolution source coding for non-difference distortion measures: Multidimensional companding," IEEE Trans. Inform. Theory, vol. 45, pp. 548-561, Mar. 1999.

[17] A. Gersho and R. M. Gray, Vector Quantization and Signal Compression. Boston, MA: Kluwer Acad. Pub., 1992.

[18] R. M. Gray and D. L. Neuhoff, "Quantization," IEEE Trans. Inform. Theory, vol. 44, pp. 2325-2383, Oct. 1998.

[19] W. R. Bennett, "Spectra of quantized signals," Bell Syst. Tech. J., vol. 27, pp. 446-472, July 1948.

[20] P. F. Panter and W. Dite, "Quantizing distortion in pulse-count modulation with nonuniform spacing of levels," Proc. IRE, vol. 39, pp. 44-48, Jan. 1951

[21] J. A. Bucklew and G. L. Wise, "Multidimensional asymptotic quantization theory with $r$ th power distortion measures," IEEE Trans. Inform. Theory, vol. IT-28, pp. 239-247, Mar. 1982.

[22] S. Cambanis and N. L. Gerr, "A simple class of asymptotically optimal quantizers," IEEE Trans. Inform. Theory, vol. IT-29, pp. 664-676, Sept. 1983.

[23] T. Linder, "On asymptotically optimal companding quantization," Prob. Contr. Inform. Theory, vol. 20, no. 6, pp. 475-484, 1991.

[24] D. L. Neuhoff, "The other asymptotic theory of lossy source coding," in Coding and Quantization (R. Calderbank, G. D. Forney, Jr., and N. Moayeri, eds.), vol. 14 of DIMACS Series in Discrete Mathematics and Theoretical Computer Science, pp. 55-65, American Mathematical Society, 1993.

[25] V. K. Goyal, "High-rate transform coding: How high is high, and does it matter?," in Proc. IEEE Int. Symp. Inform. Theory, (Sorrento, Italy), p. 207, June 2000.

[26] R. M. Gray and A. H. Gray, Jr., "Asymptotically optimal quantizers," IEEE Trans. Inform. Theory, vol. IT-23, pp. 143-144, Feb. 1977.

[27] A. Segall, "Bit allocation and encoding for vector sources," IEEE Trans. Inform. Theory, vol. IT-22, pp. 162-169, Mar. 1976. 